\title{
A Numerical Study on the Optimal design of In-line Type Subsea Separator
}

\author{
Hyunji Kim*, Youngju Kim, Namsub Woo ${ }^{\circledR}$, Sangmok Han ${ }^{\$}$, Sunchul Huh ${ }^{\star}$ \\ *Dept. of Energy Mechanical Engineering, Graduate School of Gyeongsang National University, S. Korea \\ \#@SIGAM (Korea Institute of Geoscience and Mineral Resources) \\ ${ }^{\&}$ Dept. of Energy Mechanical Engineering, Gyeongsang National University, S. Korea
}

(Received 15 May 2020, accepted 18 July 2020)

Doi: https://doi.org/10.36224/ijes.130202

\begin{abstract}
Subsea separation is an attractive and economic solution to develop deep offshore fields producing fluid without hydrate or wax. The subsea separation system should be reliable to ensure successful operation in a wide range of multiphase flow regime, without need for developments. A subsea separator can avoid or simplifying costly surface platforms of floating vessels, as well as being an efficient tool to enhance hydrocarbon production. One solution of interest is the separation and re-injection of water at the seabed to avoid bringing the water up to the surface facility. In this study, multiphase flow characteristics inside in-line type subsea separation system are investigated for the design of subsea separation system. The separation efficiency of the subsea separator is determined through experiments that are the liquid-gas phased separation. Also internal swirl element (ISE) modelling of the separator was optimized. The analysis results were utilized to improve the reliability and efficiency of the subsea separation system.
\end{abstract}

Keywords: In-line type, Separator, Optimal design, Numerical analysis, Multiphase Flow

\section{Introduction}

Generally, the offshore structure is subject to extreme loads from the harsh environmental conditions in the sea, such as waves and winds [1]. In recent years, there has been a tendency to move several production facilities, such as separators processed offshore, to the seabed recently. Subsea separators are essential equipment for subsea plants, which perform oil and gas separation processes from crude oil on the seabed [2-4].

In general, subsea separation systems are classified as gravity type, and inline type depends on the depth. Due to manufacturing cost and safety issues, it was necessary to develop a small inline type separator for the depth was over 1000m [5-7].

Slot et al., [8] discussed the flow patterns observed in newly designed separators for both single-phase and two-phase oil-water flows. Also, for the design of a small in-line subsea separation system, the multiphase flow characteristics inside the in-line subsea separator were investigated.

As the operation environment of this equipment corresponds to the deep sea or ultra-high deep sea, traditional equipment that has been previously used in offshore and onshore environments is not appropriate for this field development. Therefore, to collect oil and gas in the deep sea, equipment that is specialized for the deep sea needs to be developed [9-11]. For the equipment operated in the deep sea, it is almost impossible to reproduce an operation environment as it stands, and thus a system integration test (SIT) for actual ship/actual 
equipment is almost impossible. Using this newest simulation technology, it is possible to predict and verify the functions and dynamic behaviors of a system in advance in various conditions of the ocean and the deep sea [12-13].

In this study, a numerical analysis study was performed to analyze the multiphase flow characteristics inside an in-line subsea separation system. In particular, the separation system focuses on two-phase separation of gas-liquid mixtures. In addition, ISE modeling is optimized for separation efficiency and gas to oil ratio.

\section{Theoretical background}

In this research, governed equation used as follows.

Continuity equation:

$$
\frac{\partial}{\partial t}\left(\alpha_{q} \rho_{q}\right)+\nabla \cdot\left(\alpha_{q} \rho_{q} \overrightarrow{v_{q}}\right)=\sum_{p=1}^{n}\left(m_{p q}-m_{p q}\right)
$$

$\alpha_{q}$ and $\overrightarrow{v_{q}}$ is volume fraction and velocity of phase $\mathrm{q}, m_{p q}$ means mass transport from $p^{\text {th }}$ to $q^{\text {th }}$. Momentum equation:

$$
\begin{gathered}
\frac{\partial}{\partial t}\left(a_{q} \rho_{q} \overrightarrow{v_{q}}\right)+\nabla \cdot\left(a_{q} \rho_{q} \overrightarrow{v_{q}} \overrightarrow{v_{q}}\right)=-a_{q} \nabla p+\nabla \cdot \overline{\tau_{q}}+a_{q}+a_{q} \rho_{q} \vec{g} \\
+\sum_{p=1}^{n}\left(\vec{R}_{p q}+\dot{m}_{p q} \vec{v}_{p q}-\dot{m}_{q p} \vec{v}_{q p}\right) \\
+\left(\vec{F}_{q}+\vec{F}_{l i f t, q}+\vec{F}_{w l, q}+\vec{F}_{v m, q}+\vec{F}_{t d, q}\right)
\end{gathered}
$$

$\overline{\tau_{q}}$ is stress-strain tensor of $q^{\text {th }}$, it is defined as follows.

$$
\bar{\tau}_{q}=a_{q} \mu_{q}\left(\nabla \vec{v}_{q}+\nabla \vec{v}^{T_{a}}\right)+a_{q}\left(\lambda_{q}-\frac{2}{3} \mu_{q}\right) \nabla \cdot \vec{v}_{q} \bar{I}
$$

$\mu_{q}$ and $\lambda_{q}$ are shear and viscous of phase $\mathrm{q}, \bar{I}$ is unit tensor.

Energy Equation:

$$
\begin{aligned}
\frac{\partial}{\partial t}\left(a_{q} \rho_{q} h_{q}\right)+ & \nabla \cdot\left(a_{q} \rho_{q} \vec{u}_{q} h_{q}\right)=a_{q} \frac{\partial p_{q}}{\partial t}+\bar{\tau}_{q}: \nabla \vec{u}_{q}-\nabla \cdot \vec{q}_{q} \\
& +S_{q}+\sum_{p=1}^{n}\left(Q_{p q}+\dot{m}_{p q} h_{p q}-\dot{m}_{q p} h_{q p}\right)
\end{aligned}
$$

$h_{q}$ is specific enthalpy of $q^{\text {th }}$, and $\vec{q}_{q}$ is heat flux

\section{Finite element analysis of $1^{\text {st }}$ separator}

\subsection{Modelling}

Figure 1 is ISE modeling for numerical analysis. ISE modeling was designed with reference to previous studies [14]. The dimensions of the ISE outer diameter are $100 \mathrm{~mm}$ and other dimensions are noted in Figure 1. The pipe length is $2 \mathrm{~m}$, the pipe diameter is $100 \mathrm{~mm}$, and the ISE is fixed to the pipe. Three cases compare different wing length and tail length. The basic 
shape (Case 1) is intended to optimize the shape of the ISE for multiphase separation. Cases 1 3 are classified as vane lengths $10 \sim 20 \mathrm{~cm}$, and classifications $4 \sim 6$ are classified as tail lengths from $3 \sim 8 \mathrm{~cm}$. The ISE shape of each case is summarized in Figure 2.

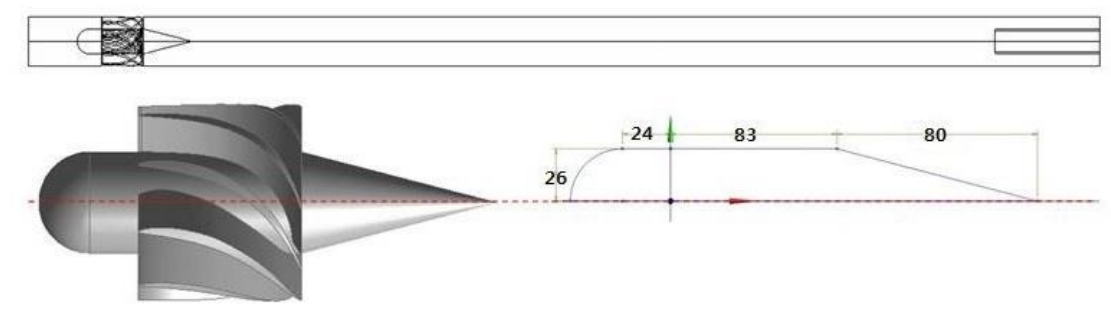

Figure 1: Initial ISE (internal swirl element) modelling

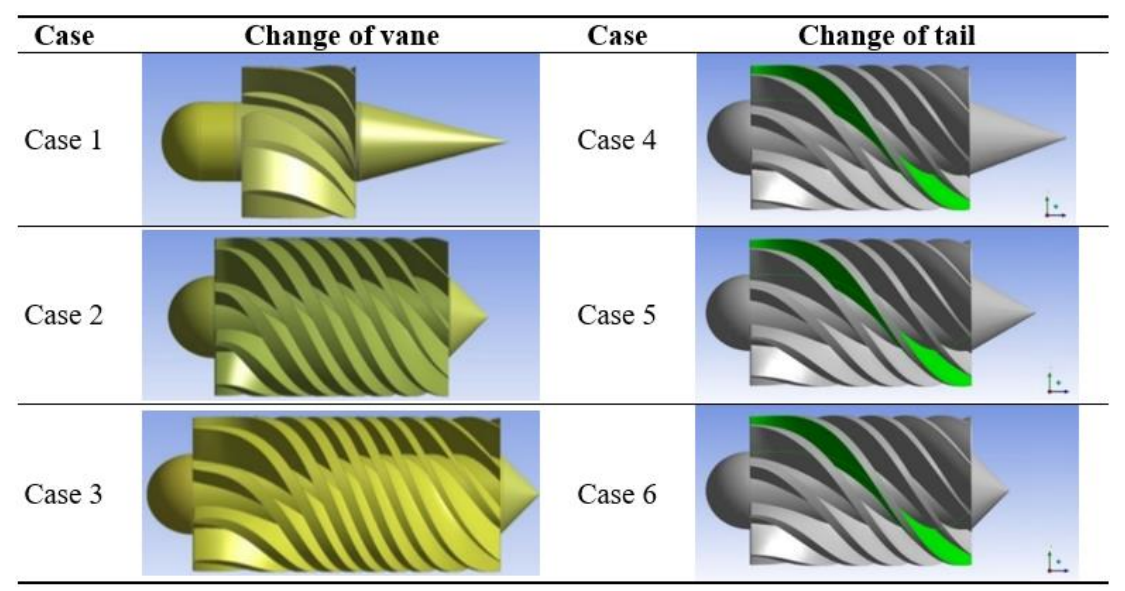

Figure 2: Case studies with the change of ISE

\subsection{Boundary condition}

The hydraulic fluid properties and boundary conditions are summarized in Table 1 . The volume ratio of the crude oil and natural gas mixture flowing at the inlet was assumed to be $80 \%$ of the oil and $20 \%$ of the gas, and the oil was set as a continuous phase and a gas as a dispersed phase. The inlet flow rate was set to $7 \mathrm{~m} / \mathrm{s}$ and the outlet to atmospheric pressure. For CFD analysis, the Eulerian model was applied due to multiphase fluid analysis. The analysis solver used version 18 of the commercial CFD code ANSYS Fluent and was analyzed with a single type separation model.

Table 1: Boundary condition of 1st separator.

\begin{tabular}{lll}
\hline \multirow{2}{*}{ Condition } & Value \\
\hline \multirow{2}{*}{ Mixture } & Liquid (water) & $80 \%$ \\
\cline { 2 - 3 } & Gas (air) & $20 \%$ \\
\hline Inlet [velocity] & $7 \mathrm{~m} / \mathrm{s}$ \\
\hline Outlet [pressure] & Atmospheric \\
\hline
\end{tabular}




\subsection{Analysis result}

As a result of numerical analysis, in all cases, water and air were separated due to the high-speed swirl flow by ISE. In addition, because of the different density, natural gas was collected in the center of the pipe, and crude oil was rotated on the outer wall of the pipe to be moved.

Figure 3 is a result of optimization analysis of the wing length of the water volume fraction contour image. Each case has a different vane length, Case 1 is $10 \mathrm{~cm}$, Case 2 is $15 \mathrm{~cm}$ and Case 3 is $20 \mathrm{~cm}$. In all cases, there is a high rate of gas in the center of the pipe and oil on the edges. The liquid volume was well maintained as an exit along the wall.

Figure 4 shows the tail length optimization analysis of the volume ratio contour image. Each case has a different tail length, Case 4 is $8 \mathrm{~cm}$, Case 5 is $5 \mathrm{~cm}$ and Case 6 is $3 \mathrm{~cm}$. In each case, a vortex is created by the tail length. Vortex directly affects the separation efficiency. As the tail length decreases, the vortex decreases and the flow is much more stable.

In Figure 5, each case of the separator calculated separation efficiency for qualitative evaluation. As a result, the maximum separation efficiency of Case 1 is $82 \%$, Case 2 is $87 \%$ and Case 3 is $93 \%$. The best model is Case 3, which is about $90 \%$ of the average separation efficiency. Therefore, the optimal design of the ISE wing length is $15 \mathrm{~cm}$ and the tail length is 3 $\mathrm{cm}$.

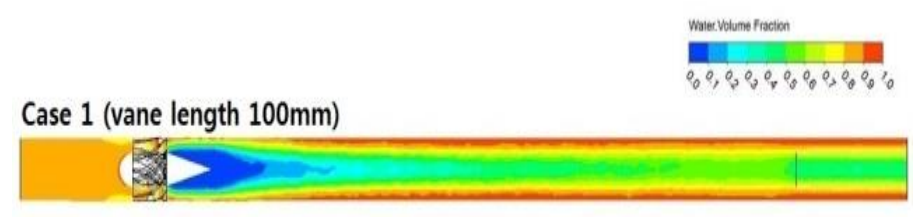

Case 2 (vane length $150 \mathrm{~mm}$ )

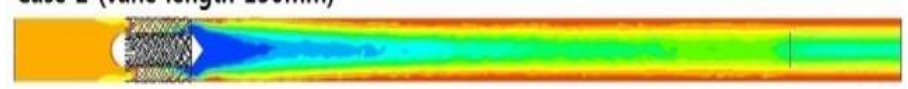

Case 3 (vane length $200 \mathrm{~mm}$ )

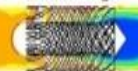

Figure 3: Analysis result of water volume fraction for compare vane length

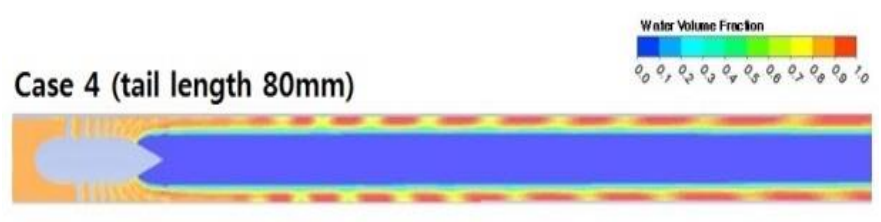

Case 5 (tail length $50 \mathrm{~mm}$ )

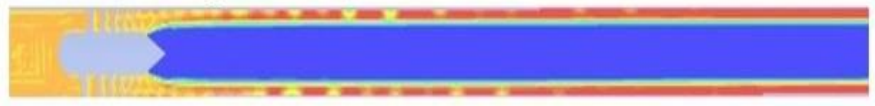

Case 6 (tail length $30 \mathrm{~mm}$ )

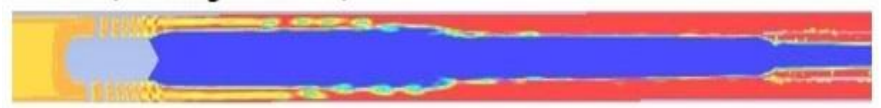

Figure 4: Analysis result of water volume fraction for compare tail length 


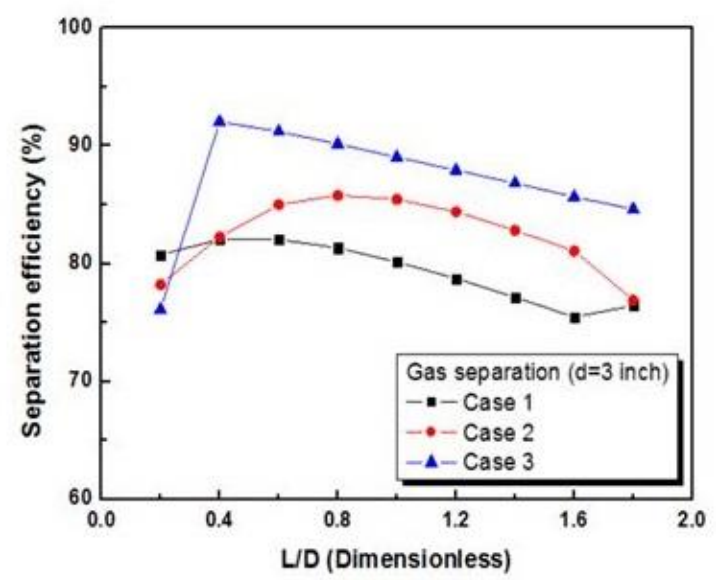

Figure 5: Separation efficiency of tail length 3 cases

\section{Finite element analysis of $2^{\text {nd }}$ separator}

\subsection{Modelling}

Figure 6 shows an optimized separator model for numerical analysis. The ISE model was optimized with reference to previous studies. The dimensions of the ISE outer diameter are 4 inches and other dimensions are noted in Figure 6. The pipe length is $2.5 \mathrm{~m}$, the pipe diameter is $2 \sim 4$ inches, and the ISE is fixed to the pipe.

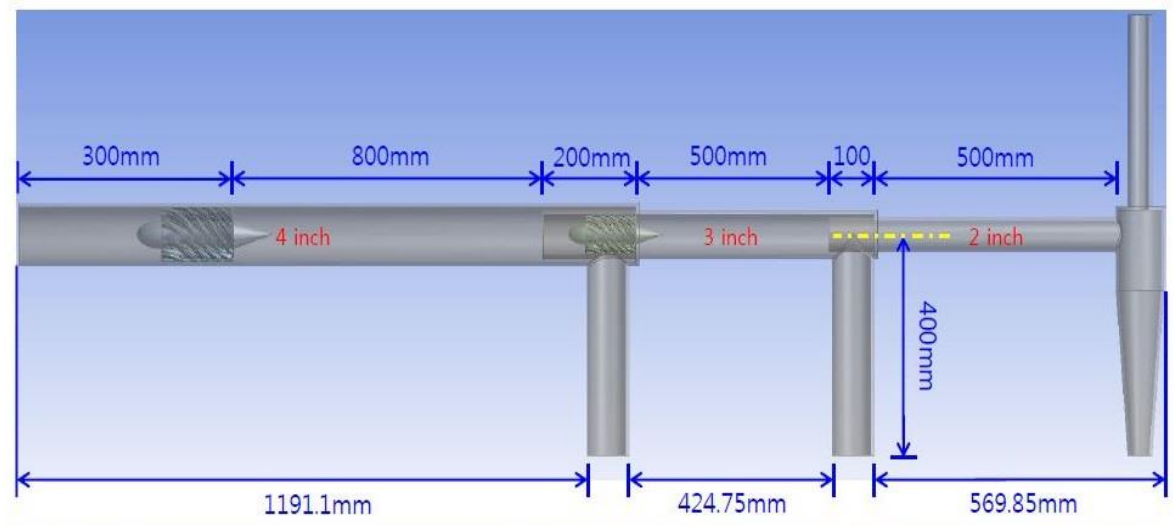

Figure 6: Optimized design of Separator

\subsection{Boundary condition}

The hydraulic fluid properties and boundary conditions are summarized in Table 2. It is assumed that the volume ratio of the crude oil and natural gas mixture flowing out of the inlet is $8 \sim 90 \%$ of the oil and $1 \sim 20 \%$ of the gas. The oil was set to the continuous phase and the gas to the dispersed phase. The inlet flow was set to $4 \sim 16 \mathrm{~L} / \mathrm{s}$, and the outlet was set to atmospheric pressure. For CFD analysis, the Eulerian model was applied due to multiphase fluid analysis. The analysis solver used the commercial CFD code ANSYS Fluent 18 version and was analyzed with a dual type separation model. 
Table 2: Boundary condition of separator.

\begin{tabular}{llll}
\hline \multicolumn{3}{l}{ Condition } & \multicolumn{2}{l}{ Value } \\
\hline \multirow{2}{*}{ Mixture } & \multicolumn{1}{l}{ Liquid (water) } & $80 \%$ & $90 \%$ \\
\cline { 2 - 3 } & Gas (air) & $20 \%$ & $10 \%$ \\
\hline Inlet [velocity] & $4 \sim 6 \mathrm{~m} / \mathrm{s}$ \\
\hline Outlet [pressure] & Atmospheric \\
\hline
\end{tabular}

\subsection{Analysis result}

As a result of numerical analysis, water and air are separated in all cases due to high-speed swirl flow by ISE. In addition, because of the different density, natural gas was collected in the center of the pipe, and crude oil was rotated on the outer wall of the pipe to be moved.

Figure 7 shows the water volume fractions of the 9:1 ratio and the 8:2 ratio, both of which retain the liquid volume well along the wall surface to the 2 nd separator. As a result, $4 \sim 6$ $\mathrm{L} / \mathrm{s}$ models have a gas volume at the first outlet due to the low flow rate. After $7 \mathrm{~L} / \mathrm{s}$, the first outlet seems to get only the volume. Each case calculates separation efficiency for qualitative evaluation.

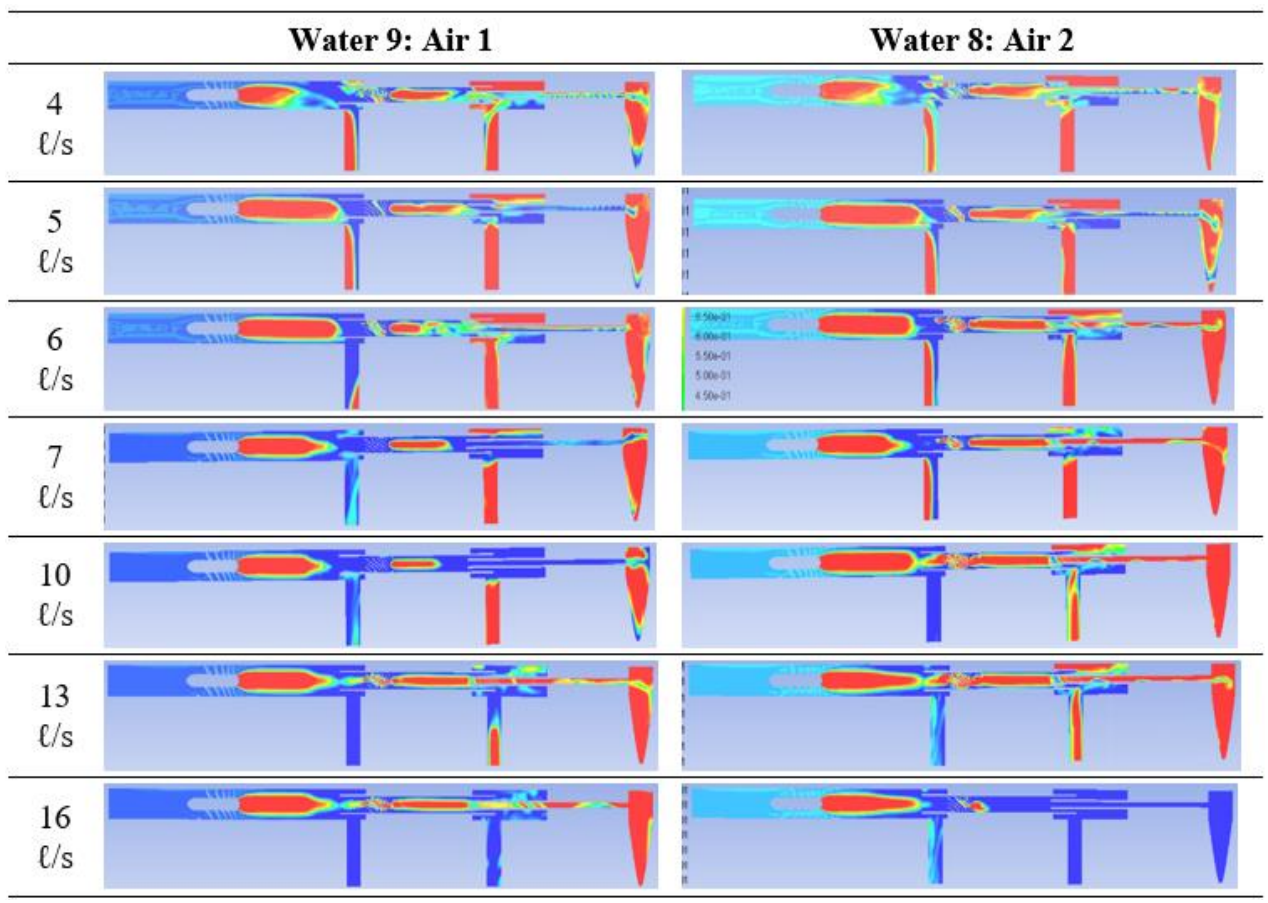

Figure 7: Analysis result of separator each case

\section{Conclusion}

In this study, the ISE of the in-line subsea separator was analyzed for shape optimization, and the numerical studies on the multiphase flow are as follows: 
Hyunji Kim et al. / International Journal of Engineering Sciences 2020 13(2) 43-49

ISE is designed for the development of in-line subsea separators for multiphase fluid separation. In the analysis, each case has a different vane length and tail length for comparison. And case 6 is the optimal design due to separation efficiency.

These results are now used to manufacture experimental devices based on optimal models. And the results of comparison between analysis and experiment are closely matched. As a result of the first exit, the separation efficiency of the 8:2 ratio was generally less than the 9:1 ratio. The $4 \sim 6 \mathrm{~L} / \mathrm{s}$ case is less efficient than the high flow case. The separation efficiency rarely increases after $7 \mathrm{~L} / \mathrm{s}$, so the average of separation efficiency in the $7 \sim 16 \mathrm{~L} / \mathrm{s}$ model is $95 \%$.

The results of this study will be used to improve the reliability and efficiency of the separation system.

\section{References}

[1] Patric A. V., Mohan P. J., Title of paper/book (italic font), Name of journal/Conference/book publisher, Volume/Edition, pp. 1- 10

[2] Devold, Havard. Oil and gas production handbook: An introduction to oil and gas production, transport, refining and petrochemical industry. ABB Oil and Gas, 2013.

[3] Khan, Faisal I., and Paul R. Amyotte. "Inherent safety in offshore oil and gas activities: a review of the present status and future directions." Journal of Loss Prevention in the Process Industries Vol.15, No.4, pp.279-289, 2002.

[4] Woo Jong Hun, Jong Ho Nam, and Kwang Hee Ko. "Development of a simulation method for the subsea production system." Journal of computational design and engineering Vol.1, No.3, pp.173186, 2014.

[5] Woo, N.S., Kim, H.J., Han, S.M., Ha, J.H., Huh, S.C. and Kim, Y.J., "Evaluation of the Structural Stability of a Heave Compensator for an Offshore Plant and the Optimization of Its Shape". Journal of nano science and nanotechnology, 20(1), pp.263-269. 2020.

[6] Kim, Youngju, et al. "Study on the Numerical Analysis of In-Line Type Subsea Separator for LiquidLiquid Mixture Flow." ASME 2018 International Mechanical Engineering Congress and Exposition. American Society of Mechanical Engineers Digital Collection, 2018.

[7] Falcone, Gioia, et al. "Multiphase flow metering: current trends and future developments." SPE annual technical conference and exhibition. Society of Petroleum Engineers, 2001.

[8] J. J. Slot, L.J.A.M. Campen, H.W.M. Hoeijmakers and, R.F. Mudde, "In-line Oil-water Separation in Swirling Flow." 8th International Conference on CFD in Oil \& Gas, Metallurgical and Process Industries SINTEF/NTNU, Trondheim NORWAY, pp.11-21, 2011.

[9] Magi, Stefano, et al. "Subsea gas-liquid separation: Case studies and technology benefits." Offshore Technology Conference. Offshore Technology Conference, 2012.

[10] Kim, Y.J., Woo, N.S., Han, S.M. and Kim, H.J., "A Study on the Separation Efficiency of In-line type Subsea Separator According to Flow Rate." Proceedings of the ASME 2017 International Mechanical Engineering Congress and Exposition (IMECE2017), November 3-9, Florida, USA, 2017.

[11] Murphy, S., Delfos, R., Pourquie, M.J.B.M., Olujic, Z., Jansens, P.J. and Nieuwstadt, F.T.M., "Prediction of strongly swirling flow within an axial hydrocyclone using two commercial CFD codes." Chemical engineering science, Vol.62, pp.1619-1635, 2007.

[12] Alary, V., F. Marchais, and T. Palermo. "Subsea water separation and injection: A solution for hydrates." Offshore Technology Conference. Offshore Technology Conference, 2000.

[13] Di Silvestro, Roberto, et al. "A Novel Gas/liquid Separator to Enhance Production of Deepwater Marginal." Offshore Technology Conference. Offshore Technology Conference, 2011.

[14] Woo, J.H., Nam, J.H. and Ko, K.H., "Development of a simulation method for the subsea production system". Journal of computational design and engineering, 1(3), pp.173-186. 2014 